\title{
AXIOMS CONCERNING UNCERTAIN DISAGREEMENT POINTS IN 2-PERSON BARGAINING PROBLEMS
}

\section{Youngsub Chun}

Seoul National University, Korea

ychunesnu.ac.kr

\begin{abstract}
We consider 2-person bargaining situations in which the feasible set is known, but the disagreement point is uncertain. We investigate the implications of various axioms concerning uncertain disagreement points and characterize the family of linear solutions, which includes the egalitarian, lexicographic egalitarian, Nash, and Kalai-Rosenthal solutions. We also show that how the important subfamilies (or members) of this family can be singled out by imposing additional axioms or strengthening the axioms used in the characterizations.
\end{abstract}

Keywords: Axiomatic approach to bargaining problems, uncertain disagreement point, linear solutions.

JEL Classification Numbers: C71, C78, D70.

\section{INTRODUCTION}

A formulated by Nash (1950), the bargaining problem consists of a feasible $A$ set and a disagreement point, represented in utility space. The agents can achieve any point in the feasible set if they agree on it. Otherwise, they end up at the disagreement point. We are interested in the existence of solutions for such problems which satisfy a certain set of axioms. ${ }^{1}$

This is a revised version of my paper circulated as "Axioms concerning uncertain disagreement points for 2-person bargaining problems" (Working Paper No.99, University of Rochester) and "The role of uncertain disagreement points in 2-person bargaining" (Discussion Paper No. 87-17, Southern Illinois University). I am grateful to William Thomson, Hans Peters, Shiran Rachmilevitch, and Ching-jen Sun for their comments.

1 For a survey of the literature, see Thomson $(1994,1998)$.

Copyright (C) Youngsub Chun / 6(1), 2021, 37-58. 
In the traditional formulation, it is assumed that the disagreement point is fixed. The possibility of a varying disagreement point has also been the subject of a number of articles (Thomson, 1987; Livne, 1986, 1989; Peters, 2010; Bossert, 1994; Rachmilevitch, 2011a, 2011b; Anbarci \& Sun, 2013). Moreover, bargaining situations in which the feasible set is known but the disagreement point is uncertain have been studied extensively (Chun, 1989, 1990; Chun \& Thomson, 1990a, 1990b, 1990c; Livne, 1988, 1989; Peters \& van Damme, 1991). This paper is also focused on bargaining situations in which the feasible set is known, but the disagreement point is uncertain.

Suppose that bargaining takes place today, without precise knowledge of the location of the disagreement point, this uncertainty being resolved tomorrow. Under what conditions can agents reach an agreement today? A minimal requirement is that each agent should be guaranteed at least the minimum of what she receives when the uncertainty is lifted tomorrow. Otherwise, the agent is definitely better off by waiting until tomorrow. We require that all agents should be guaranteed this minimum. This requirement of disagreement point quasi-concavity was introduced in Chun \& Thomson (1990b) and variants are studied by Chun \& Thomson (1990a), Livne (1988), Peters (2010), and Peters $\&$ van Damme (1991). The purpose of this paper is to explore the implication of this axiom for 2-person bargaining problems.

We provide characterizations of the family of linear solutions studied by Chun (1990). They are defined as follows. Let $\delta$ be a function associating with each problem a non-negative direction. Moreover, if another problem with the same feasible set has a disagreement on the line passing through the disagreement point of the first problem in the direction assigned by the function $\delta$, then that problem has the same direction $\delta$. The linear solution relative to $\delta$ is defined by choosing as a solution outcome of each problem at the maximal feasible point such that the vector of utility gains from the disagreement point is in the direction determined by applying $\delta$ to the problem. This family of solutions, which we call the linear family, is fairly large, including many well-known solutions such as the egalitarian, lexicographic egalitarian, Nash, and Kalai-Rosenthal solutions.

By imposing disagreement point quasi-concavity in conjunction with the standard conditions of weak Pareto optimality, individual rationality, and continuity (with respect to the disagreement point and the feasible set), we characterize the continuous members of the linear family. Also, by strengthening weak Pareto optimality to Pareto optimality and weakening continuity (to 
continuity with respect to the disagreement point), we characterize the Pareto optimal members of the family. Other characterizations of the family can be obtained by using axioms related to disagreement point quasi-concavity. We also show how well-known subfamilies or members of the family can be singled out by imposing additional axioms or strengthening the axioms used in the characterizations.

The paper is organized as follows. Section 2 contains some preliminaries and introduces the basic axioms. Section 3 states our main axiom of disagreement point quasi-concavity and characterizes the linear family. Section 4 discusses axioms related to disagreement point quasi-concavity and establishes alternative characterizations of the linear family. Finally, Section 5 characterizes various subfamilies including the egalitarian, lexicographic egalitarian, Nash, and Kalai-Rosenthal solutions.

\section{PRELIMINARIES}

Let $N=\{1,2\}$ be the set of agents. A 2-person bargaining problem, or simply a problem, is a pair $(S, d)$, where $S$ is a subset of $\mathbb{R}^{2}$ and $d$ is a point in $S$ such that

(1) $S$ is convex and closed,

(2) $a_{i}(S)=\max \left\{x_{i} \mid x=\left(x_{1}, x_{2}\right) \in S\right\}$ exists for all $i \in N$,

(3) $S$ is comprehensive, i.e., for all $x \in S$ and all $y \in \mathbb{R}^{N}$, if ${ }^{2} y \leq x$, then $y \in S$,

(4) there exists $x \in S$ such that $x>d$.

The set $S$ is the feasible set. Each point $x$ of $S$ is a feasible alternative. The coordinates $x \in S$ are the von Neumann-Morgenstern utility levels attained by the agents through the choice of some joint action. The point $d$ is the disagreement point (or "status quo"). The intended interpretation of $(S, d)$ is as follows: the agents can achieve any point in $S$ if they unanimously agree on

2 Vector inequalities: given $x, y \in \mathbb{R}^{N}, x \geqq y$ means $x_{i} \geq y_{i}$ for all $i \in N, x \geq y$ means $x \geqq y$ and $x \neq y, x>y$ means $x_{i}>y_{i}$ for all $i \in N$. 
it. If they do not agree on any point, they end up at $d$. Let $\Sigma^{2}$ be the class of all problems and $\Gamma^{2}$ be the class of all feasible sets satisfying (1), (2) and (3).

A solution is a function $F: \Sigma^{2} \rightarrow \mathbb{R}^{2}$ such that for all $(S, d) \in \Sigma^{2}$, $F(S, d) \in S$. The value taken by the solution $F$ when applied to the problem $(S, d), F(S, d)$, is the solution outcome of $(S, d)$.

Now we introduce standard axioms in the literature. Weak Pareto optimality requires that there should be no feasible alternative at which all agents are strictly better off than at the solution outcome. Pareto optimality requires that the solution outcome should exhaust all gains from cooperation.

Weak Pareto optimality (WPO): For all $(S, d) \in \Sigma^{2}$ and all $x \in \mathbb{R}^{2}$, if $x>$ $F(S, d)$, then $x \notin S$.

Pareto optimality (PO): For all $(S, d) \in \Sigma^{2}$ and all $x \in \mathbb{R}^{2}$, if $x \geq F(S, d)$, then $x \notin S$.

Let $W P O(S)=\left\{x \in S \mid\right.$ for all $x^{\prime} \in \mathbb{R}^{2}, x^{\prime}>x$ implies $\left.x^{\prime} \notin S\right\}$ be the set of weakly Pareto optimal points of $S$. Similarly, let $P O(S)=\{x \in S \mid$ for all $x^{\prime} \in \mathbb{R}^{2}, x^{\prime} \geq x$ implies $\left.x^{\prime} \notin S\right\}$ be the set of Pareto optimal points of $S$.

Individual rationality requires that no agent should be worse off at the solutions outcome than at the disagreement point. Strong individual rationality requires that all agents should be strictly better off than at the disagreement point.

Individual rationality $(I R)$ : For all $(S, d) \in \Sigma^{2}, F(S, d) \geqq d$.

Strong individual rationality (SIR): For all $(S, d) \in \Sigma^{2}, F(S, d)>d$.

Let $\operatorname{IR}(S, d)=\{x \in S \mid x \geqq d\}$ be the set of individually rational points of $(S, d)$.

Next are two continuity properties. $d$-continuity (respectively, $S$-continuity) requires that a small change in the disagreement point (respectively, the feasible set) cause only a small change in the solution outcome.

$d$-continuity (d-CONT): For all sequences $\left\{\left(S^{k}, d^{k}\right)\right\} \subset \Sigma^{2}$ and all $(S, d) \in \Sigma^{2}$, if $S^{k}=S$ for all $k$ and $d^{k} \rightarrow d$, then $F\left(S^{k}, d^{k}\right) \rightarrow F(S, d)$.

In the following, convergence of a sequence of sets is evaluated in the Hausdorff topology. 
$S$-continuity (S-CONT): For all sequences $\left\{\left(S^{k}, d^{k}\right)\right\} \subset \Sigma^{2}$ and all $(S, d) \in \Sigma^{2}$, if $S^{k} \rightarrow S$ and $d^{k}=d$ for all $k$, then $F\left(S^{k}, d^{k}\right) \rightarrow F(S, d)$.

Our final two axioms require that the solution outcome should dominate or be dominated by a reference point. In mid-point domination 1, the reference point is the average of the disagreement point and the maximal feasible utility level of each agent guaranteeing the other agent the utility level at the disagreement point. In mid-point domination 2, the reference point is the average of the disagreement point and the maximal feasible utility level of each agent.

Mid-point domination 1 (MPD1): For all $(S, d) \in \Sigma^{2}, F(S, d) \geqq \frac{d+a(S, d)}{2}$, where for all $i \in N, a_{i}(S, d)=\max \left\{x_{i} \mid x \in \operatorname{IR}(S, d)\right\}$.

Mid-point domination 2 (MPD2): For all $(S, d) \in \Sigma^{2}, F(S, d) \geqq \frac{d+a(S)}{2}$ or $F(S, d) \leqq \frac{d+a(S)}{2}$.

The following notation and terminology will be used frequently. Given $x_{1}, \ldots, x_{k} \in \mathbb{R}^{2}, \operatorname{comp}\left\{x_{1}, \ldots, x_{k}\right\}$ is the comprehensive hull of these points $x$ (the smallest comprehensive set containing them). Given $A \subset \mathbb{R}^{2}, \operatorname{Int}(A)$ is the relative interior of A. $\Delta^{n-1}$ is the $(n-1)$-dimensional simplex. Given $x \in \mathbb{R}^{2}$ and $\delta \in \Delta^{1}, \ell(x, \delta)$ is the line passing through $x$ in the direction $\delta$. Finally, given $x, y \in \mathbb{R}^{2}$ such that $x \neq y, \ell(x, y)$ is the line passing through $x$ and $y$.

\section{DISAGREEMENT POINT QUASI-CONCAVITY. THE MAIN CHARACTERIZATION}

The main objective of this paper is to explore the implication of the following axiom introduced by Chun \& Thomson (1990b).

Disagreement point quasi-concavity (D. $Q-C A V)$ : For all $\left(S^{1}, d^{1}\right),\left(S^{2}, d^{2}\right) \in \Sigma^{2}$, all $i \in N$, and all $\alpha \in[0,1]$. if $S^{1}=S^{2}=S$, then $F_{i}\left(S, \alpha d^{1}+(1-\alpha) d^{2}\right) \geq$ $\min \left\{F_{i}\left(S, d^{1}\right), F_{i}\left(S, d^{2}\right)\right\}$.

Note that $\left(S, \alpha d^{1}+(1-\alpha) d^{2}\right)$ is a well-defined element of $\Sigma^{2}$.

This axiom can be motivated on the basis of timing of bargaining. To illustrate, consider two agents who will face one of two equally likely problems 
$\left(S, d^{1}\right)$ and $\left(S, d^{2}\right)$ tomorrow, having the same feasible set but different disagreement points. The agents have two options: either they wait until tomorrow for the uncertainty to be lifted and solve then whatever problem has come up, or they consider the problem obtained by taking as a disagreement point the average of $d^{1}$ and $d^{2}$ and solve that problem today. If for some agent $i, F_{i}\left(S, \frac{d^{1}+d^{2}}{2}\right)$ is smaller than the minimum of $F_{i}\left(S, d^{1}\right)$ and $F_{i}\left(S, d^{2}\right)$, then the agent will definitely prefer waiting. For agent $i$ to be persuaded that the problem should be solved today, she should be guaranteed at least the minimum of $F_{i}\left(S, d^{1}\right)$ and $F_{i}\left(S, d^{2}\right)$. D.Q-CAV provides this minimum incentive to all agents.

We are interested in the following family of solutions (Chun, 1990), which generalizes the egalitarian, lexicographic egalitarian, Nash, and Kalai-Rosenthal solutions.

Definition Let $\delta: \Sigma^{2} \rightarrow \Delta^{1}$ be a function such that for all $S \in \Gamma^{2}$ and all $y \in \operatorname{Int}(S), y \in \ell(d, \delta(S, d))$ implies that $\delta(S, y)=\delta(S, d)$ and is continuous with respect to $y$. The linear solution relative to $\delta, F^{\delta}$, is defined by setting, for all $(S, d) \in \Sigma^{2}, F^{\delta}(S, d)$ equal to $\ell(d, \delta(S, d)) \cap W P O(S)$.

Note that for $F^{\delta}$ to be well-defined, it should be that for all $S \in \Gamma^{2}$ and all $d^{1}$, $d^{2} \in \operatorname{Int}(S)$, if $\delta\left(S, d^{1}\right) \neq \delta\left(S, d^{2}\right)$, then $\ell\left(d^{1}, \delta\left(S, d^{1}\right)\right) \cap \ell\left(d^{2}, \delta\left(S, d^{2}\right)\right) \cap$ $\operatorname{Int}(S)=\emptyset$.

We now turn to the results. The proof of Lemma 1 is the same as the proof of Lemma 1 in Chun \& Thomson (1990b).

Lemma 1. Let $F$ be a solution satisfying WPO, IR, and D.Q-CAV. Also, let $(S, d) \in \Sigma^{2}$ be such that $F(S, d) \in P O(S)$. Then, for all $x \in[d, F(S, d)[$, $F(S, x)=F(S, d)$.

Proof. First, note that for all $x \in[d, F(S, d)],(S, x) \in \Sigma^{2}$. Let $x \in$ $[d, F(S, d)]$ be given. Let $\bar{\lambda} \in[0,1]$ be such that $x=\bar{\lambda} d+(1-\bar{\lambda}) F(S, d)$, and $\left\{\lambda^{k}\right\} \subset[0,1]$ be such that $\lambda^{k}<\bar{\lambda}$ for all $k$ and $\lambda^{k} \rightarrow \bar{\lambda}$. Also, let $x^{k}=\frac{x-\lambda^{k} d}{1-\lambda^{k}}$ for all $k$. Then, $\left(S, x^{k}\right) \in \Sigma^{2}$ for all $k$. By D.Q-CAV, $F_{i}(S, x) \geq$ $\min \left\{F_{i}\left(S, x^{k}\right), F_{i}(S, d)\right\}$ for all $i$ and all $k$. As $k \rightarrow \infty, x^{k} \rightarrow F(S, d)$ and since $F(S, d) \in P O(S)$, it follows from IR that $F\left(S, x^{k}\right) \rightarrow F(S, d)$. Therefore, $F(S, x) \geqq F(S, d)$. Since $F(S, d) \in P O(S)$, we conclude that $F(S, x)=F(S, d)$. 
Lemma 2. Let $F$ be a solution satisfying WPO, IR, $d$-CONT, and D.Q-CAV. Also, let $(S, d) \in \Sigma^{2}$ be such that $F(S, d) \in \operatorname{Int}(P O(S))$. Then, for all $x \in \ell(d, F(S, d)) \cap \operatorname{Int}(S), F(S, x)=F(S, d)$.

Proof. Let $F$ and $(S, d) \in \Sigma^{2}$ satisfying the hypothesis of the lemma be given. From Lemma 1, we know that for all $x \in[d, F(S, d)], F(S, x)=F(S, d)$. Now, suppose by way of contradiction that there exists $y \in \operatorname{Int}(S)$ such that $d \in[y, F(S, d)]$ and $F(S, y) \neq F(S, d)$. Since $F(S, d) \in \operatorname{Int}(P O(S))$, from WPO and $d$-CONT, we can assume that $F(S, y) \in \operatorname{Int}(P O(S))$.

(a) We consider the case when $\ell(d, F(S, d))$ is neither horizontal nor vertical. Suppose that $F_{1}(S, y)>F_{1}(S, d)$. Let $z=\left(y_{1}, d_{2}\right)$.

Claim 1. $F_{1}(S, z) \leq F_{1}(S, d)$.

Otherwise, from WPO and $d$-CONT, there exists $\left.z^{1} \in\right] z, d[$ such that $F_{1}(S, d)<F_{1}\left(S, z^{1}\right) \leq F_{1}(S, z)$ and $F\left(S, z^{1}\right) \in P O(S)$. From Lemma 1, for all $x \in\left[z^{1}, F\left(S, z^{1}\right)\right], F(S, x)=F\left(S, z^{1}\right)$. Since $F_{2}\left(S, z^{1}\right) \geq z_{2}^{1}=d^{2}$ by IR, there exists $\bar{x} \in[d, F(S, d)] \cap\left[z^{1}, F\left(S, z^{1}\right)\right]$ which is a contradiction.

Claim 2. $F_{1}(S, y)>F_{1}(S, d)$ is impossible.

Since $F_{1}(S, z) \leq F_{1}(S, d)$, by $d$-CONT, there exists $z^{2} \in[z, y]$ such that $F\left(S, z^{2}\right)=F(S, d)$. From Lemma 1 , for all $x \in\left[z^{2}, F(S, d)\right], F(S, x)=$ $F(S, d)$. Also, from WPO, IR and Lemma 1, for all $x \in\left[z^{2}, d\right], F(S, x)=$ $F(S, d)$. Now define the sequence of problems $\left\{\left(S, z^{k}\right)\right\}$ by setting $z^{k+1}=$ $\frac{1}{2}\left(z^{k}+y\right)$ for all $k \geq 2$. Also, for all $k \geq 3$, let $\ell^{k}$ be the line passing through $z^{k}$ and $d$, and $a^{k}=\ell^{k} \cap W P O(S)$. For all $\left.\left.x \in\right] z^{2}, z^{3}\right]$, if $F_{1}(S, d)<F_{1}(S, x) \leq$ $\min \left\{F_{1}(S, y), a_{1}^{3}\right\}$, then there exists $z^{\prime}$ such that $z^{\prime} \in \ell(x, F(S, x)) \cap \ell\left(z^{2}, d\right)$. Since we assumed that $F(S, x) \neq F(S, d)$, this is impossible. Therefore, for all $\left.x \in] z^{2}, z^{3}\right]$, we have $F_{1}(S, x) \leq F_{1}(S, d)$ or $F_{1}(S, x)>\min \left\{F_{1}(S, y), a_{1}^{3}\right\}$. By $d$-CONT, we have $F_{1}(S, x) \leq F_{1}(S, d)$ for all $x \in\left[z^{2}, z^{3}\right]$. Repeating the same procedure, for all $x \in\left[z^{2}, y\left[\right.\right.$, we obtain $F_{1}(S, x) \leq F_{1}(S, d)$. Therefore, $F_{1}(S, y)>F_{1}(S, d)$ contradicts $d$-CONT.

By a similar argument, we obtain a contradiction to $F_{1}(S, y)<F_{1}(S, d)$.

(b) Now suppose that $\ell(d, F(S, d))$ is horizontal and there exists $y \in \operatorname{Int}(S)$ such that $d \in[y, F(S, d)]$ and $F(S, y) \neq F(S, d)$. By IR and $d$-CONT, there exists $z^{1} \in\left[y, d\left[\right.\right.$ such that $F\left(S, z^{1}\right) \neq F(S, d)$ and $\ell\left(z^{1}, F\left(S, z^{1}\right)\right)$ is positively sloped. From (a), for all $z \in \ell\left(z^{1}, F\left(S, z^{1}\right)\right) \cap \operatorname{Int}(S), F(S, z)=F\left(S, z^{1}\right)$. 


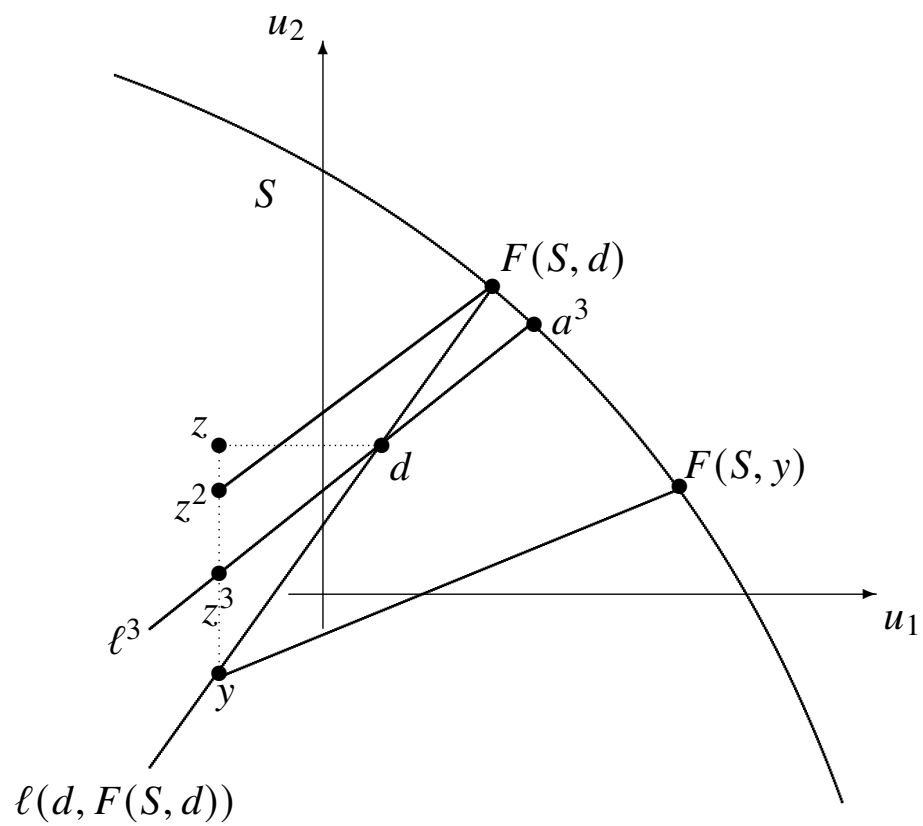

Figure 1: Proof of Claim 2 in Lemma 2.

Now let $a^{*}$ be the Pareto optimal point of $S$ on the line passing through $d$ parallel to $\ell\left(z^{1}, F\left(S, z^{1}\right)\right)$. For some $z \in\left[z^{1}, d\right]$, say $z^{2}$, if $\ell\left(z^{2}, F\left(S, z^{2}\right)\right)$ is flatter than $\ell\left(z^{1}, F\left(S, z^{1}\right)\right)$, then there exists $z^{\prime} \in \operatorname{Int}(S)$ such that $z^{\prime} \in$ $\ell\left(z^{1}, F\left(S, z^{1}\right)\right) \cap \ell\left(z^{2}, F\left(S, z^{2}\right)\right)$, which is impossible. Therefore, for all $z \in\left[z^{1}, d\right], F_{1}(S, z)<a_{1}^{*}$. This is incompatible with $d$-CONT. A similar argument can be established when $\ell(d, F(S, d))$ is vertical.

Remark 3. Lemma 1 can easily be generalized to $n$-person problems. However, it remains an open question whether Lemma 2 can be generalized to such problems.

Now we present our main results.

Theorem 4. A solution satisfies PO, IR, $d$-CONT, and D.Q-CAV if and only if it is a linear solution $F^{\delta}$ with the additional property that for all $(S, d) \in \Sigma^{2}$, $\ell(d, \delta(S, d)) \cap W P O(S) \backslash P O(S)=\emptyset$. 
Proof. It is obvious that all $F^{\delta}$ satisfy IR, $d$-CONT and D.Q-CAV, and if $\delta$ satisfies the additional property, PO. Conversely, let $F$ be a solution satisfying the four axioms. For all $(S, d) \in \Sigma^{2}$, let $\delta(S, d)=\frac{F(S, d)-d}{\|F(S, d)-d\|}$. Since PO and IR together imply that $F(S, d) \geq d, \delta$ is a well-defined function from $\Sigma^{2}$ to $\Delta^{1}$. It is enough to show that for all $\left(S^{1}, d^{1}\right),\left(S^{2}, d^{2}\right) \in \Sigma^{2}$, if $S^{1}=S^{2}=S$ and $d^{2} \in \ell\left(d^{1}, F\left(S, d^{1}\right)\right)$, then $\delta\left(S, d^{2}\right)=\delta\left(S, d^{1}\right)$. If $F\left(S, d^{1}\right) \in \operatorname{Int}(P O(S))$, then the desired conclusion follows from Lemma 2. Suppose now that $F\left(S, d^{1}\right) \notin \operatorname{Int}(P O(S))$ and that $\delta\left(S, d^{1}\right) \neq \delta\left(S, d^{2}\right)$. From Lemma 1, for all $d \in\left[d^{1}, F\left(S, d^{1}\right)\right], F(S, d)=F\left(S, d^{1}\right)$ and for all $d \in\left[d^{2}, F\left(S, d^{2}\right)\right], F(S, d)=F\left(S, d^{2}\right)$. By PO and $d$-CONT, there exists $d^{\prime} \in$ $\left[d^{1}, d^{2}\right]$ such that $F\left(S, d^{\prime}\right) \in \operatorname{Int}(P O(S)), F\left(S, d^{\prime}\right) \neq F\left(S, d^{2}\right)$ and that either $\ell\left(d^{\prime}, F\left(S, d^{\prime}\right)\right) \cap\left[d^{1}, F\left(S, d^{1}\right)\right] \neq \emptyset$ or $\ell\left(d^{\prime}, F\left(S, d^{\prime}\right)\right) \cap\left[d^{2}, F\left(S, d^{2}\right)\right] \neq \emptyset$. Since $F\left(S, d^{\prime}\right) \neq F\left(S, d^{1}\right)$ and $F\left(S, d^{\prime}\right) \neq F\left(S, d^{2}\right)$, it is a contradiction.

Finally, we note that PO implies that for all $(S, d) \in \Sigma^{2}, \ell(d, \delta(S, d)) \cap$ $W P O(S) \backslash P O(S)=\emptyset$.

Remark 5. The family of solutions characterized in Theorem 4 is fairly large, including the lexicographic egalitarian, Nash, and Kalai-Rosenthal solutions. However, the egalitarian solution is excluded, since it violates PO.

Theorem 6. A solution satisfies WPO, IR, $d$-CONT, $S$-CONT, and D.Q-CAV if and only if it is a linear solution $F^{\delta}$ with the additional property that $\delta$ is continuous with respect to $S$.

Proof. It is obvious that all $F^{\delta}$ satisfy WPO, IR, $d$-CONT, and D.Q-CAV, and if $\delta$ is continuous with respect to $S, S$-CONT. Conversely, let $F$ be a solution satisfying the five axioms. For all $(S, d) \in \Sigma^{2}$, let $\delta(S, d)=\frac{F(S, d)-d}{\|F(S, d)-d\|}$. Since WPO and IR together imply that $F(S, d) \geq d, \delta$ is a well-defined function from $\Sigma^{2}$ to $\Delta^{1}$. It is enough to show that for all $(S, d) \in \Sigma^{2}$, if there exists $d^{\prime} \in$ $\ell(d, F(S, d)) \cap \operatorname{Int}(S)$, then $\delta\left(S, d^{\prime}\right)=\delta(S, d)$. If $F(S, d) \in \operatorname{Int}(P O(S))$, then the desired conclusion follows from Lemma 2. Otherwise, let $\left\{\left(S^{k}, d\right)\right\} \subset \Sigma^{2}$ be a sequence of problems such that for all $k, F\left(S^{k}, d\right) \in \operatorname{Int}\left(P O\left(S^{k}\right)\right)$ and $d \in \operatorname{Int}\left(S^{k}\right)$ and that $S^{k} \rightarrow S$. By the previous argument, $F\left(S^{k}, d\right)=F^{\delta}\left(S^{k}, d\right)$ for all $k$, and by $S$-CONT, $F(S, d)=F^{\delta}(S, d)$.

Finally, we note that $S$-CONT implies the continuity of $\delta(\cdot, x)$ with respect to $S$ in the Hausdorff topology.

Remark 7. The family of solutions characterized in Theorem 6 includes the egalitarian, Nash, and Kalai-Rosenthal solutions. However, the lexicographic egalitarian solution is excluded, since it violates $S$-CONT. 


\section{VARIANTS OF THE MAIN RESULT}

Bargaining situations in which the feasible set is known but the disagreement point is uncertain have been studied extensively in the literature. Moreover, several axioms related to D.Q-CAV have appeared. Here we discuss how the linear family can be characterized using these axioms.

The first axiom, which we call weak disagreement point linearity, was introduced by Livne (1988) in his study of the Nash solution. ${ }^{3}$

Weak disagreement point linearity (W.D.LIN): For all $\left(S^{1}, d^{1}\right),\left(S^{2}, d^{2}\right) \in \Sigma^{2}$ and all $\alpha \in[0,1]$, if $S^{1}=S^{2}=S$ and $F\left(S, d^{1}\right)=F\left(S, d^{2}\right)=x$, then $F\left(S, \alpha d^{1}+(1-\alpha) d^{2}\right)=x$.

This axiom can be interpreted on the basis of timing of bargaining. To see this, consider two agents who will face one of two equally likely problems $\left(S, d^{1}\right)$ and $\left(S, d^{2}\right)$ tomorrow, having the same feasible set, but different disagreement points. Suppose that the solution outcome of the two problems coincides. Since all agents receive the same amount tomorrow irrespective of the uncertainty, it is natural to require that they should receive the same amount when the problem is solved today by taking as a disagreement point the average of $d^{1}$ and $d^{2}$. W.D.LIN provides such an incentive to agents.

Now we explore the implication of this axiom for 2-person bargaining problems. By replacing D.Q-CAV by W.D.LIN in Theorems 4 and 6, we obtain the same conclusions. In addition, by using the following weak condition, a characterization of the linear family can be established.

Boundary (BOUND): For all sequences $\left\{\left(S^{k}, d^{k}\right)\right\} \subset \Sigma^{2}$ and all $(S, d) \in \Sigma^{2}$, if $S^{k}=S$ for all $k, F(S, d)=x$ and $d^{k} \rightarrow x$, then $F\left(S^{k}, d^{k}\right) \rightarrow x$.

For a solution satisfying Pareto optimality, BOUND is just a considerable weakening of IR. For a solution satisfying only WPO, BOUND is a continuity property which requires that if the disagreement point is closer to the boundary of the feasible set, then the solution outcome is also closer to the disagreement point. It is a weak condition satisfied by all well-known solutions.

Now we have the following result.

3 Under the name of independence of convex combination of equivalent conflict outcomes. 
Lemma 8. Let $F$ be a solution satisfying WPO, IR, $d$-CONT, BOUND, and W.D.LIN. Also, let $(S, d) \in \Sigma^{2}$ be given. Then, for all $x \in[d, F(S, d)[$, $F(S, x)=F(S, d)$.

Proof. First, note that for all $x \in\left[d, F(S, d)\left[,(S, x) \in \Sigma^{2}\right.\right.$. We assume that $W P O(S)$ contains a vertical segment. The case when $W P O(S)$ contains a horizontal (or both a vertical and horizontal) segment can be dealt with similarly. Now suppose by way of contradiction that there exists $d^{1} \in[d, F(S, d)]$ such that $F\left(S, d^{1}\right) \neq F(S, d)$. Two cases are possible:

(i) $F_{2}\left(S, d^{1}\right)>F_{2}(S, d)$.

Note that if $F_{2}\left(S, d^{1}\right)>F_{2}(S, d)$, IR implies that $\ell(d, F(S, d))$ is not vertical. Let $d^{2} \in \operatorname{Int}(S)$ be such that $d_{1}^{2}=d_{1}$ and that for all $a \in \operatorname{IR}\left(S, d^{2}\right), a_{2}>$ $F_{2}\left(S, d^{1}\right)$. By WPO, $F\left(S, d^{2}\right) \in W P O(S)$ and by $\operatorname{IR}, F_{2}\left(S, d^{2}\right)>F_{2}\left(S, d^{1}\right)$. By $d$-CONT, there exists $d^{3} \in\left[d^{2}, d\right]$ such that $F\left(S, d^{3}\right)=F\left(S, d^{1}\right)$. By W.D.LIN, for all $d^{\prime} \in\left[d^{1}, d^{3}\right], F\left(S, d^{\prime}\right)=F\left(S, d^{1}\right)$.

Now let $d(\lambda)$ be a parametrization of $\left[d^{1}, F(S, d)\right]$ such that $d(0)=d^{1}$ and $d(1)=F(S, d)$. By $d$-CONT, $F(S, d(\lambda))$ moves continuously. By BOUND, there exists $\bar{\lambda} \in[0,1]$ such that $F_{2}\left(S, d^{1}\right)>F_{2}(S, d(\bar{\lambda})) \geq F_{2}(S, d)$. Let $d(\bar{\lambda})=d^{4}$. Also, by $d$-CONT, there exists $d^{5} \in\left[d^{3}, d\right]$ such that $F\left(S, d^{5}\right)=F\left(S, d^{4}\right)$. By W.D.LIN, for all $d^{\prime} \in\left[d^{4}, d^{5}\right], F\left(S, d^{\prime}\right)=F\left(S, d^{4}\right)$. Then, $\left[d^{1}, d^{3}\right]$ and $\left[d^{4}, d^{5}\right]$ intersect. Let $d^{6}$ be the intersection point. Clearly, $d^{6} \in \operatorname{Int}(S)$. Since $F\left(S, d^{1}\right) \neq F\left(S, d^{4}\right)$, it is a contradiction.

(ii) $F_{2}\left(S, d^{1}\right)<F_{2}(S, d)$.

From the same argument as in (i), for all $d^{\prime} \in\left[d^{1}, F\left(S, d^{1}\right)\left[, F_{2}\left(S, d^{\prime}\right) \leq\right.\right.$ $F_{2}\left(S, d^{1}\right)$. Let $d^{2}$ be a point in $] d^{1}, F\left(S, d^{1}\right)[$.

Let $d(\lambda)$ be a parametrization of $\left[d^{2}, F(S, d)\right]$ such that $d(0)=d^{2}$ and $d(1)=F(S, d)$. By $d$-CONT, $F(S, d(\lambda))$ moves continuously. By BOUND, there exists $\bar{\lambda} \in\left[0,1\left[\right.\right.$ such that $F_{2}(S, d) \geq F_{2}(S, d(\bar{\lambda}))>F_{2}\left(S, d^{1}\right)$. Let $d(\bar{\lambda})=d^{3}$. Also, by $d$-CONT, there exists $d^{4} \in\left[d^{2}, d\right]$ such that $F\left(S, d^{4}\right)=$ $F\left(S, d^{3}\right)$. By W.D.LIN, for all $d^{\prime} \in\left[d^{3}, d^{4}\right], F\left(S, d^{\prime}\right)=F\left(S, d^{3}\right)$. Then, $\left[d^{1}, F\left(S, d^{1}\right)\right]$ and $\left[d^{3}, d^{4}\right]$ intersect. Let $d^{5}$ be the intersection point. Clearly, $d^{5} \in \operatorname{Int}(S)$. Since $d^{5} \in\left[d^{1}, F\left(S, d^{1}\right)\right], F_{2}\left(S, d^{5}\right) \leq F_{2}\left(S, d^{1}\right)$, and since $d^{5} \in\left[d^{3}, d^{4}\right], F_{2}\left(S, d^{5}\right)=F_{2}\left(S, d^{3}\right)>F_{2}\left(S, d^{1}\right)$. This is a contradiction.

Theorem 9. A solution satisfies WPO, IR, $d$-CONT, BOUND, and W.D.LIN if and only if it is a linear solution. 
Proof. It is obvious that all $F^{\delta}$ satisfy the five axioms. Conversely, let $F$ be a solution satisfying the five axioms. First, we know from Lemma 8 that for all $(S, d) \in \Sigma^{2}$, and all $x \in[d, F(S, d)], F(S, x)=F(S, d)$. It remains to extend the conclusion of Lemma 8 to all $x \in \ell(d, F(S, d)) \cap \operatorname{Int}(S)$. Since the proof is similar to that of Lemma 2, we omit it.

The second axiom was introduced by Peters \& van Damme (1991) in their study of the Nash solution. ${ }^{4}$

Disagreement point linearity (D.LIN): For all $(S, d) \in \Sigma^{2}$ and all $\alpha \in[0,1]$, $F(S, \alpha d+(1-\alpha) F(S, d))=F(S, d)$.

Let $(S, d)$ be given and consider a new problem obtained by taking the same feasible set and a different disagreement point, which is a convex combination of the old disagreement point and its solution outcome. Then, D. LIN, a strengthening of W.D.LIN, requires that the solution outcome be the same in two problems. If we extend our domain of bargaining problems to allow the disagreement point to lie on the boundary of the feasible set and define the solution outcome of such problems be the disagreement point, then the motivation similar to W.D.LIN can be given.

Now we explore the implication of this axiom for 2-person problems. Again, by replacing D.Q-CAV by D.LIN in Theorems 4 and 6, we obtain the same conclusion. In addition, the following theorem can be established.

Theorem 10. A solution satisfies WPO, IR, $d$-CONT, and D.LIN if and only if it is a linear solution.

Proof. It is obvious that all $F^{\delta}$ satisfy the four axioms. The converse statement can be established by exploiting the logical implications of these axioms. Indeed, it can easily be shown that (i) WPO and D.LIN together imply W.D.LIN, and that (ii) $d$-CONT and D.LIN together imply BOUND. Therefore, by Theorem 9, we obtain the desired conclusion.

Remark 11. If IR is dropped from the list in Theorem 10, then the following generalized linear solutions are permissible. Let $B^{1}=\left\{x \in \mathbb{R}^{2}\left|\sum\right| x_{i} \mid=1\right.$ and $\left.-x \notin \mathbb{R}_{+}^{2}\right\}$ and given $x \in \mathbb{R}^{2}$ and $\delta \in B^{1}$, let $\bar{\ell}(d, \delta)$ be the line passing through $d$ in the direction $\delta$. Also, given $(S, d) \in \Sigma^{2}$, let $\bar{\ell}(d, \delta) \cap W P O(S)$

${ }^{4}$ Under the name of convexity. 
be the weakly Pareto optimal point of $S$ on the half-line passing through $d$ in the direction $\delta$.

Definition Let $\delta$ be a function such that, for all $(S, d) \in \Sigma^{2}, \delta(S, d) \in B^{1}$ and that for all $S \in \Gamma^{2}$ and all $y \in \operatorname{Int}(S), y \in \bar{\ell}(d, \delta(S, d))$ implies that $\delta(S, y)=\delta(S, d)$ and that $\delta(S, \cdot)$ is continuous with respect to $d$. Given the function $\delta$, the generalized linear solution relative to $\delta$ is defined by setting for each $(S, d) \in \Sigma^{2}, F^{\delta}(S, d)$ equal to $\bar{\ell}(d, \delta(S, d)) \cap W P O(S)$.

\section{FURTHER CHARACTERIZATIONS}

In this section, we discuss how important subfamilies of the linear family can be characterized by imposing additional axioms or strengthening the axioms used in the Theorems 4 and 6.

\subsection{The Egalitarian Solution}

First, we consider a subfamily of the linear family, which generalizes the well-known egalitarian solution (Kalai, 1977; Thomson \& Myerson, 1980).

Definition. Given a continuous function $\delta: \Gamma^{2} \rightarrow \Delta^{1}$, the directional solution relative to $\delta, E^{\delta}$, is defined by setting for all $(S, d) \in \Sigma^{2}, E^{\delta}(S, d)$ equal to $\ell(d, \delta(S)) \cap W P O(S)$. Given $\alpha \in \Delta^{1}$, the weighted egalitarian solution with weights $\alpha, E^{\alpha}$, is defined by setting for all $(S, d) \in \Sigma^{2}, E^{\alpha}(S, d)$ equal to $\ell(d, \alpha) \cap W P O(S)$. The egalitarian solution is obtained by choosing $\alpha_{1}=\alpha_{2}$.

This family can be characterized by the following axiom, which strengthens D.Q-CAV.

Disagreement point concavity (D.CAV): For all $\left(S^{1}, d^{1}\right),\left(S^{2}, d^{2}\right) \in \Sigma^{2}$ and all $\alpha \in[0,1]$, if $S^{1}=S^{2}=S$, then $F\left(S, \alpha d^{1}+(1-\alpha) d^{2}\right) \geqq \alpha F\left(S, d^{1}\right)+(1-$ $\alpha) F\left(S, d^{2}\right)$.

This axiom, introduced and studied in Chun \& Thomson (1990a), gives an even stronger incentive to all agents to reach an agreement today than D.Q-CAV does. To illustrate, consider two agents who will face one of two equally likely problems $\left(S, d^{1}\right)$ and $\left(S, d^{2}\right)$ tomorrow, having the same feasible set, but different disagreement points. The agents have two options: either they 
wait until tomorrow for the uncertainty to be lifted and solve then whatever problem has come up, or they consider the problem obtained by taking as a disagreement point the average of $d^{1}$ and $d^{2}$ and solve that problem today. The expected payoff associated with the contingent agreements of the first option is $\frac{F\left(S, d^{1}\right)+F\left(S, d^{2}\right)}{2}$ and that associated with the second option is $F\left(S, \frac{d^{1}+d^{2}}{2}\right)$, since $\frac{d^{1}+d^{2}}{2}$ is the corresponding "expected" disagreement point. If either $F\left(S, \frac{d^{1}+d^{2}}{2}\right)$ weakly dominates $\frac{F\left(S, d^{1}\right)+F\left(S, d^{2}\right)}{2}$ or the reverse holds, all agents agree on when to do. A conflict may arise if neither of these inequalities holds. If a solution satisfies D.CAV, then all agents agree to solve the problem today.

It can easily be checked that D.CAV implies D.Q-CAV. D.CAV can be regarded as a dual to an axiom considered by Myerson (1981) concerning uncertainty in the feasible set (variants of which are studied by Perles \& Maschler (1981), Peters (1986), and Chun (1988)).

The following result, which can be generalized to $n$-person bargaining problems, is due to Chun \& Thomson (1990a). We note that $d$-CONT is not needed.

Theorem 12. (Chun \& Thomson, 1990a) A solution satisfies WPO, IR, $S$ CONT, and D.CAV if and only if it is a directional solution.

The family of weighted egalitarian solutions can be characterized by strengthening IR to the following axiom.

Independence of Non-Individually Rational Alternatives (INIR): For all $(S, d) \in$ $\Sigma^{2}, F(S, d)=F(\operatorname{comp}\{\operatorname{IR}(S, d)\}, d)$.

This axiom, introduced by Peters (2010), says that the non-individually rational alternatives are irrelevant to the determination of the solutions outcome. It is a natural condition since agents are guaranteed their utilities at the disagreement point. It can easily be checked that WPO, INIR and S-CONT (or PO and INIR) together imply IR.

Theorem 13. (Chun \& Thomson, 1990a) A solution satisfies WPO, INIR, $S$-CONT, and D.CAV if and only if it is a weighted egalitarian solution.

Alternative characterizations of the weighted egalitarian solutions can be found in Chun \& Thomson (1990a, 1990c). In particular, they show that the weighted egalitarian solutions can be characterized by additionally imposing the following axiom of contraction independence (Nash, 1950) ${ }^{5}$ to Theorem

5 Under the name of independence of irrelevant alternatives. 
Contraction independence (CI): For all $\left(S^{1}, d^{1}\right),\left(S^{2}, d^{2}\right) \in \Sigma^{2}$, if $S^{2} \subseteq S^{1}$, $d^{2}=d^{1}$, and $F\left(S^{1}, d^{1}\right) \in S^{2}$, then $F\left(S^{2}, d^{2}\right)=F\left(S^{1}, d^{1}\right)$.

CI requires that if an alternative has been judged superior to all others in some feasible set, then it should be judged superior to all others in any subset (to which it belongs) provided the disagreement point is kept constant.

Theorem 14. (Chun \& Thomson, 1990a, 1990c) A solution satisfies WPO, IR, $S$-CONT, D.CAV, and CI if and only if is a weighted egalitarian solution.

The egalitarian solution is the only weighted egalitarian solution satisfying the following axiom.

Symmetry $(S Y)$ : For all $(S, d) \in \Sigma^{2}$ and all permutations $\pi:\{1,2\} \rightarrow\{1,2\}$, if $S=\pi(S)$ and $d=\pi(d)$, then $F_{1}(S, d)=F_{2}(S, d)$.

SY says that if the only information available on the conflict situation is contained in the mathematical description of $(S, d)$, and $(S, d)$ is a symmetric problem, then there is no ground for favoring one agent at the expense of another.

Corollary 15. (Chun \& Thomson, 1990a, 1990c) A solution satisfies WPO, INIR, S-CONT, D.CAV, and SY (or WPO, IR, S-CONT, D.CAV, CI, and SY) if and only if it is the egalitarian solution.

Let $\tilde{\Sigma}^{2}$ be the class of problems satisfying (1), (2) and (3) which allows the disagreement point to be on the boundary of the problem. On $\tilde{\Sigma}^{2}$, Bossert \& Peters (2021) provide an alternative characterization of the weighted egalitarian solutions by weakening D.CAV to individual disagreement point concavity, dropping $S$-CONT, and additionally imposing translation invariance and disagreement point sensitivity. Translation invariance requires that adding a constant to an agent's utility function should change the solution outcome by the constant. Disagreement point sensitivity requires that the solution outcome should respond to certain changes in the disagreement point. Finally, individual disagreement point concavity requires that the conclusion of D.CAV should hold if there is only one agent whose utility at the disagreement point is uncertain. 
Translation invariance (T.INV): For all $(S, d) \in \Sigma^{2}$ and all $b \in \mathbb{R}^{2}, F(S+b, d+$ b) $=F(S, d)+b$.

Disagreement point sensitivity (D.SEN): For all $(S, d),\left(S, d^{\prime}\right) \in \Sigma^{2}$ such that $d \nsupseteq d^{\prime}, F(S, d) \neq F\left(S, d^{\prime}\right)$.

Individual disagreement point concavity (I.D.CAV): For all $\left(S^{1}, d^{1}\right),\left(S^{2}, d^{2}\right) \in$ $\Sigma^{2}$, all $i \in N$, and all $\alpha \in[0,1]$, if $S^{1}=S^{2}=S$ and for all $j \in N \backslash\{i\}, d_{j}^{1}=d_{j}^{2}$, then $F_{i}\left(S, \alpha d^{1}+(1-\alpha) d^{2}\right) \geq \alpha F_{i}\left(S, d^{1}\right)+(1-\alpha) F_{i}\left(S, d^{2}\right)$.

Theorem 16. (Bossert \& Peters, 2021) A solution satisfies WPO, T.INV, IR, IIA, D.SEN, and I.D.CAV if and only if is a weighted egalitarian solution.

Once again, the egalitarian solution can be characterized by additionally imposing SY to the list appearing in Theorem 16.

\subsection{The Lexicographic Egalitarian Solution}

The directional solutions often violate PO. The following extension, called the lexicographic egalitarian solution, ${ }^{6}$ is an adaptation of the egalitarian solution that satisfies PO. On the other hand, this solution does not satisfy $S$-CONT.

Definition. The lexicographic egalitarian solution, $L$, is defined by setting, for all $(S, d) \in \Sigma^{2}, L(S, d)=E(S, d)$ if $E(S, d) \in P O(S)$ and $L(S, d)=\{x \in$ $P O(S) \mid x_{1}=E_{1}(S, d)$ or $\left.x_{2}=E_{2}(S, d)\right\}$, otherwise.

Chun \& Thomson (1990a) showed that no solution satisfies PO, IR and D.CAV together. However, the following weakening of D.CAV is compatible with PO and IR (Chun, 1990).

Restricted disagreement point concavity (R.D.CAV): For all $\left(S^{1}, d^{1}\right),\left(S^{2}, d^{2}\right) \in$ $\Sigma^{2}$ and all $\alpha \in[0,1]$, if $S^{1}=S^{2}=S$ and $F\left(S, d^{1}\right), F\left(S, d^{2}\right) \in \operatorname{Int}(P O(S))$, then $F\left(S, \alpha d^{1}+(1-\alpha) d^{2}\right) \geqq \alpha F\left(S, d^{1}\right)+(1-\alpha) F\left(S, d^{2}\right)$.

The motivation for this axiom is same as for D.CAV, except that the conclusion is required to hold for the interior of the Pareto optimal set. For a solution satisfying PO, if it chooses the boundary point of the Pareto optimal set as the solution outcome, then the solution outcome becomes less sensitive to changes

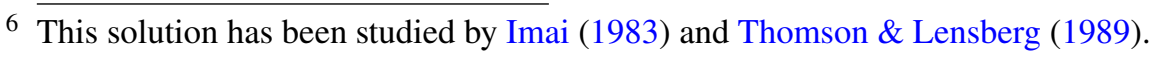


in the disagreement point. Therefore, it is unreasonable to require that the solution behave well even on the boundary. It can easily be checked that PO, $d$-CONT and R.D.CAV (or PO, IR and R.D.CAV) together imply D.Q-CAV.

To characterize the lexicographic egalitarian solution, $S$-CONT is weakened to the following condition.

Pareto-continuity $(P-C O N T)$ : For all sequences $\left\{\left(S^{k}, d^{k}\right)\right\} \subset \Sigma^{2}$ and all $(S, d) \in$ $\Sigma^{2}$, if $S^{k} \rightarrow S, P O\left(S^{k}\right) \rightarrow P O(S)$, and $d^{k}=d$ for all $k$, then $F\left(S^{k}, d^{k}\right) \rightarrow$ $F(S, d)$.

P-CONT requires that a small change in the feasible set and the Pareto optimal set cause only a small change in the solution outcomes. It can easily be checked that $S$-CONT implies P-CONT.

Now we are ready to present a characterization of the lexicographic egalitarian solution.

Theorem 17. (Chun, 1989) A solution satisfies PO, SY, INIR, $d$-CONT, P-CONT, and R.D.CAV if and only if it is the lexicographic egalitarian solution.

\subsection{The Nash Solution}

Now we discuss the best-known solution in the axiomatic bargaining theory, the Nash solution. This solution, introduced and characterized by Nash (1950), has been extensively studied in the literature. Properties which describe its behavior with respect to changes in the disagreement point have been investigated by Chun \& Thomson (1990b), Peters (2010), and Peters \& van Damme (1991).

Definition. Given $\alpha \in \operatorname{Int}\left(\Delta^{1}\right)$, the weighted Nash solution with weights $\alpha$, $N^{\alpha}$, is defined by setting for all $(S, d) \in \Sigma^{2}, N^{\alpha}(S, d)$ to be the maximizer of the product $\Pi\left(x_{i}-d_{i}\right)^{\alpha_{i}}$ over $\operatorname{IR}(S, d)$. The Nash solution, $N$, is the member of this family obtained by choosing $\alpha_{1}=\alpha_{2}$.

To characterize the Nash solution, we introduce an invariance property. A positive affine transformation is a function $\lambda: \mathbb{R}^{2} \rightarrow \mathbb{R}^{2}$ given by $a \in \mathbb{R}_{++}^{2}$ and $b \in \mathbb{R}^{2}$ such that for all $x \in \mathbb{R}^{2}, \lambda(x)=\left(a_{1} x_{1}+b_{1}, a_{2} x_{2}+b_{2}\right)$.

Scale invariance $(S . I N V)$ : For all $(S, d) \in \Sigma^{2}$ and all positive affine transformations $\lambda: \mathbb{R}^{2} \rightarrow \mathbb{R}^{2}, F(\lambda(S), \lambda(d))=\lambda(F(S, d))$. 
S.INV can be justified by the fact that agents' utility functions are von NeumannMorgenstern types, which are unique up to positive affine transformations. Of course, S.INV implies T.INV.

Nash (1950) showed that his solution is the unique solution satisfying PO, SY, CI, and S.INV. Now we establish an alternative characterization of the Nash solution by investigating logical implications of CI and other axioms.

Lemma 18. Let $F$ be a continuous linear solution characterized in Theorem 6 . Then, the solution satisfies CI if and only if it satisfies INIR.

Proof. It is clear that for a solution satisfying IR, CI implies INIR. To prove the converse statement, let $\left(S^{1}, d\right),\left(S^{2}, d\right) \in \Sigma^{2}$ be two problems such that $S^{2} \subseteq S^{1}$ and $F\left(S^{1}, d\right) \in S^{2}$. Now define the sequence of problems $\left\{\left(S^{k}, d^{k}\right)\right\}$ such that $S^{2} \subseteq S^{k} \subseteq S^{1}, S^{k} \rightarrow S^{2}, d^{k} \in\left[d, F\left(S^{1}, d\right)\right]$ and $\operatorname{IR}\left(S^{k}, d^{k}\right)=\operatorname{IR}\left(S^{1}, d^{k}\right)$ for all $k$. By INIR, $F\left(S^{k}, d^{k}\right)=F\left(S^{1}, d^{k}\right)$ for all $k$. Since $d^{k} \in[d, F(S, d)]$ and $F$ belongs to the linear family, $F\left(S^{k}, d^{k}\right)=F\left(S^{k}, d\right)$ and $F\left(S^{1}, d^{k}\right)=F\left(S^{1}, d\right)$ for all $k$. Altogether, we have $F\left(S^{k}, d\right)=F\left(S^{1}, d\right)$ for all $k$. Since $F$ is continuous, we conclude that $F\left(S^{2}, d\right)=F\left(S^{1}, d\right)$.

Variants of the following theorem can be found in Chun \& Thomson (1990b), Peters (2010), and Peters \& van Damme (1991). Note that $S$-CONT and S.INV together imply $d$-CONT.

Theorem 19. (Chun \& Thomson, 1990b) A solution satisfies PO, INIR, $S$-CONT, D.Q-CAV, and S.INV if and only if it is a weighted Nash solution. ${ }^{7}$

Remark 20. By dropping $S$-CONT form Theorem 19, the following solutions are permissible.

Definition. Given $i$, the $i^{\text {th }}$ benevolent dictatorial solution, $D^{i}$, is defined by setting for all $(S, d) \in \Sigma^{2}, D^{i}(S, d)$ equal to the point of $\operatorname{IR}(S, d) \cap P O(S)$ preferred by agent $i$.

In fact, we can show that a solution satisfies PO, INIR, $d$-CONT, D.Q-CAV and S.INV if and only if it is a weighted Nash solution or a benevolent dictatorial solution. Since its proof is similar to that of Theorem 1 in Peters (2010), we omit it.

7 Note that the Nash solution does not satisfy D.Q-CAV in the bargaining problem with more than 2-agents, as discussed in Chun \& Thomson (1990b). 
Remark 21. D.Q-CAV in Theorem 19 can be replaced by the following axiom:

Restricted disagreement point linearity (R.D.LIN): For all $\left(S^{1}, d^{1}\right),\left(S^{2}, d^{2}\right) \in$ $\Sigma^{2}$ and all $\alpha \in[0,1]$, if $S^{1}=S^{2}=S, \alpha F\left(S, d^{1}\right)+(1-\alpha) F\left(S, d^{2}\right) \in P O(S)$, and $S$ is smooth at both $F\left(S, d^{1}\right)$ and $F\left(S, d^{2}\right)$, then $F\left(S, \alpha d^{1}+(1-\alpha) d^{2}\right)=$ $\alpha F\left(S, d^{1}\right)+(1-\alpha) F\left(S, d^{2}\right)$.

This result can be generalized to $n$-person bargaining problems. For details, we refer to Chun \& Thomson (1990b).

On the other hand, Peters \& van Damme (1991) characterize the weighted Nash solutions by imposing D.LIN.

Theorem 22. (Peters \& van Damme, 1991) A solution satisfies SIR, INIR, $d$-CONT, S.INV, and D.LIN if and only if it is a weighted Nash solution.

It is well-known that the Nash solution is the only weighted Nash solution satisfying the symmetry axiom.

Corollary 23. (Chun \& Thomson, 1990b; Peters \& van Damme, 1991) A solution satisfies PO, INIR, S-CONT, D.Q-CAV, S,INV and SY (or SIR, INIR, $d$-CONT, S,INV, D.LIN, and SY) if and only if it is the Nash solution.

An alternative characterization of the Nash solution can be obtained by imposing MPD1.

Theorem 24. (Chun, 1990) A solution satisfies WPO, $d$-CONT, S-CONT, D.Q-CAV, and MPD1 if and only if it is the Nash solution.

Equivalently, Theorem 24 can be understood as stating that among the continuous linear solutions characterized in Theorem 6, the Nash solution is the only one satisfying MPD1. Also, Peters (2010) shows how the Nash solution can be singled out from the linear family.

\subsection{The Kalai-Rosenthal Solution}

Finally, we discuss the Kalai \& Rosenthal (1978) solution.

Definition. The Kalai-Rosenthal solution, $K R$, is defined by setting, for all $(S, d) \in \Sigma^{2}, K R(S, d)$ be the maximal point of $S$ on the line segment connecting $d$ and $a(S)$, where for each $i, a_{i}(S)=\max \left\{x_{i} \mid x \in S\right\}$. 
To characterize the Kalai-Rosenthal solution, we introduce two additional axioms. For all $(S, d) \in \Sigma^{2}$, let $T\left(S_{d}\right)=\operatorname{comp}\left\{\left(d_{1}, a_{2}(S)\right),\left(a_{1}(S), d_{2}\right)\right\}$.

Independence of strongly individually rational outcome (ISIR): For all $(S, d) \in$ $\Sigma^{2}$ and all $x \in \mathbb{R}^{2}$, if $S=\operatorname{comp}\{\operatorname{IR}(S, d)\}, x \leq d$ and $F\left(T\left(S_{d}\right), x\right)=$ $F\left(T\left(S_{d}\right), d\right)$, then $F(S, x)=F(S, d)$.

Strict disagreement point monotonicity (S.D.MON): For all $\left(S^{1}, d^{1}\right),\left(S^{2}, d^{2}\right) \in$ $\Sigma^{2}$ and all $i, j$ such that $i \neq j$, if $S^{1}=S^{2}, d_{i}^{1}=d_{i}^{2}, d_{j}^{1}<d_{j}^{2}$, and $a(S) \notin S$, then $F_{j}\left(S^{2}, d^{2}\right)>F_{j}\left(S^{1}, d^{1}\right)$.

ISIR, introduced by Peters (2010), is interpreted as a weak form of path independence. S.D.MON, introduced by Livne (1989), requires that if an agent's utility at the disagreement point increases while the other's remains fixed, then the agent should gain strictly. This is a strengthening of the condition introduced by Thomson (1987).

The next theorem follows from Peters (2010).

Theorem 25. A solution satisfies PO, IR, $d$-CONT, D.Q-CAV, SY, S.INV, ISIR, and S.D.MON if and only if it is the Kalai-Rosenthal solution.

Proof. It is clear that KR satisfies all eight axioms. Conversely, let $F$ be a solution satisfying the eight axioms. From Theorem 4, it is a Pareto-optimal member of the linear solution. Now by borrowing the proof of Theorem 2 in Peters (2010), we can obtain the desired conclusion. ${ }^{8}$

An alternative characterization of the Kalai-Rosenthal solution can be obtained by imposing MPD2.

Theorem 26. (Chun, 1990) A solution satisfies WPO, $d$-CONT, $S$-CONT, D.Q-CAV, and MPD2 if and only if it is the Kalai-Rosenthal solution.

As in Theorem 24, Theorem 26 can be understood as stating that among the continuous linear solutions characterized in Theorem 6, the Kalai-Rosenthal solution is the only one satisfying MPD2. Therefore, it is interesting to note that among the linear solutions, the Nash and Kalai-Rosenthal solutions can be singled out by imposing two different mid-point domination conditions.

$8 \overline{\text { It remains to check whether all axioms }}$ are independent. 


\section{References}

Anbarci, N., \& Sun, C. (2013). Robustness of intermediate agreements and bargaining solutions. Games and Economic Behavior, 77, 367-376.

Bossert, W. (1994). Disagreement point monotonicity, transfer responsiveness, and the egalitarian bargaining solution. Social Choice and Welfare, 11, 381-392.

Bossert, W., \& Peters, H. (2021). Individual disagreement point concavity and the bargaining problem. International Journal of Economic Theory, Forthcoming.

Chun, Y. (1988). Nash solution and timing of bargaining. Economics Letters, 28, 27-31.

Chun, Y. (1989). Lexicographic egalitarian solution and uncertainty in the disagreement point. Zeitschrift für Operations Research, 33, 259-266.

Chun, Y. (1990). Minimal cooperation in bargaining. Economics Letters, 34, 311-316.

Chun, Y., \& Thomson, W. (1990a). Bargaining with uncertain disagreement points. Econometrica, 58(4), 951-959.

Chun, Y., \& Thomson, W. (1990b). Nash solution and uncertain disagreement points. Games and Economic Behavior, 2, 213-223.

Chun, Y., \& Thomson, W. (1990c). Egalitarian solutions and uncertain disagreement points. Economics Letters, 33, 29-33.

Imai, H. (1983). Individual monotonicity and lexicographic maxmin solution. Econometrica, 51, 389-401.

Kalai, E. (1977). Proportional solutions to bargaining situations: Interpersonal utility comparisons. Econometrica, 45, 1623-1630.

Kalai, E., \& Rosenthal, R. (1978). Arbitration of two-party disputes under ignorance. International Journal of Game Theory, 7, 65-72.

Livne, Z. (1986). The bargaining problem: Axioms concerning changes in the conflict point. Economics Letters, 21, 131-134.

Livne, Z. (1988). The bargaining problem with an uncertain conflict outcome. Mathematical Social Sciences, 15, 287-302.

Livne, Z. (1989). On the status quo sets induced by the Raiffa solution to the two-person bargaining problem. Mathematics of Operations Research, 14, 688-692.

Myerson, R. B. (1981). Utilitarianism, egalitarianism, and the timing effect in social choice problems. Econometrica, 49, 883-897.

Nash, J. F. (1950). The bargaining problem. Econometrica, 18, 155-162.

Perles, M. A., \& Maschler, M. (1981). The super-additive solution for the Nash bargaining game. International Journal of Game Theory, 10, 163-193.

Peters, H. (1986). Simultaneity of issues and additivity in bargaining. Econometrica, 54, 153-169.

Peters, H. (2010). Characterizations of bargaining solutions by properties of their 
status quo sets. In A. V. Deeman \& A. Rusinowska (Eds.), Collective Decision Making: Views from Social Choice and Welfare (p. 231-247). Springer.

Peters, H., \& van Damme, E. (1991). Characterizing the Nash and Raiffa bargaining solutions by disagreement point axioms. Mathematics of Operations Research, 16, 447-461.

Rachmilevitch, S. (2011a). Disagreement point axioms and the egalitarian bargaining solution. International Journal of Game Theory, 40(1), 63-85.

Rachmilevitch, S. (2011b). A characterization of the Kalai-Smorodinsky bargaining solution by disagreement point monotonicity. International Journal of Game Theory, 40(4), 691-696.

Thomson, W. (1987). Monotonicity of bargaining solutions with respect to the disagreement point. Journal of Economic Theory, 42, 50-58.

Thomson, W. (1994). Cooperative models of bargaining. In R. J. Aumann \& S. Hart (Eds.), Handbook of Game Theory with Economic Applications (Vol. 2). North-Holland.

Thomson, W. (1998). Bargaining Theory: The Axiomatic Approach. (Forthcoming.) Academic Press.

Thomson, W., \& Lensberg, T. (1989). Axiomatic Theory of Bargaining with a Variable Number of Agents. Cambridge, U.K.: Cambridge University Press.

Thomson, W., \& Myerson, R. (1980). Monotonicity and independence axioms. International Journal of Game Theory, 9, 37-49. 